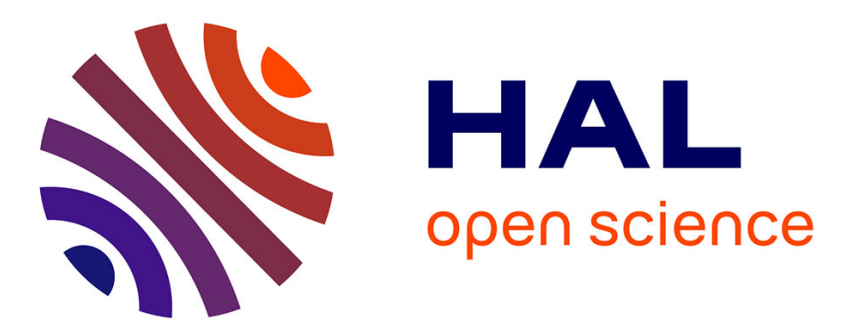

\title{
Qui ne vient pas chercher ses résultats après un dépistage positif d'une IST en CeGIDD?
}

Camille Rolland, Christophe Segouin, Elise de La Rochebrochard, Marc Shelly, Pénélope Troude

\section{- To cite this version:}

Camille Rolland, Christophe Segouin, Elise de La Rochebrochard, Marc Shelly, Pénélope Troude. Qui ne vient pas chercher ses résultats après un dépistage positif d'une IST en CeGIDD ?. Médecine et Maladies Infectieuses, 2018, 48 (Suppl. 4), pp.S99. 10.1016/j.medmal.2018.04.249 . hal-02366887

\section{HAL Id: hal-02366887 \\ https://hal.science/hal-02366887}

Submitted on 16 Nov 2019

HAL is a multi-disciplinary open access archive for the deposit and dissemination of scientific research documents, whether they are published or not. The documents may come from teaching and research institutions in France or abroad, or from public or private research centers.
L'archive ouverte pluridisciplinaire HAL, est destinée au dépôt et à la diffusion de documents scientifiques de niveau recherche, publiés ou non, émanant des établissements d'enseignement et de recherche français ou étrangers, des laboratoires publics ou privés. 
Rolland Camille, Segouin Christophe, La Rochebrochard Elise (de), Shelly Marc, Troude Pénélope, 2018, «Qui ne vient pas chercher ses résultats après un dépistage positif d'une IST en CeGIDD ? », Médecine et Maladies Infectieuses, 48, Suppl. 4, p. S99. DOI: 10.1016/j.medmal.2018.04.249.

\title{
Qui ne vient pas chercher ses résultats après un dépistage positif d'une IST en CeGIDD?
}

\author{
C. Rolland ${ }^{1}$, C. Segouin ${ }^{1}$, E. de Larochebrochard ${ }^{2}$, M. Shelly ${ }^{3}$, P. Troude ${ }^{1}$ \\ ${ }^{1}$ Service de santé publique, HU Fernand-Widal, AP-HP, Paris, France \\ ${ }^{2}$ Institut national d'études démographiques, Paris, France \\ ${ }^{3}$ CeGIDD, HU Fernand-Widal, Paris, France
}

\section{Introduction}

Malgré des avancées notables en France, près de 6000 séropositivités VIH ont été découvertes en 2015 et les infections sexuellement transmissibles (IST) sont en recrudescence. Aussi, le dépistage et le traitement précoces sont des éléments clés pour assurer un meilleur contrôle des épidémies. Le nonretour en consultation de rendu des résultats en CeGIDD (centre gratuit de dépistage) représente une perte de chance dans les approches préventive et curative, particulièrement pour les patients dépistés positifs. L'objectif de cette étude était de rechercher les facteurs associés au non-retour des consultants dépistés positifs pour le VIH, une hépatite virale ou une IST (syphilis, chlamydia ou gonocoque) en consultation de rendu de résultats dans les 30 jours suivant un dépistage en CeGIDD.

\section{Matériels et méthodes}

Les consultants dépistés positifs lors d'un dépistage réalisé au CeGIDD entre octobre 2016 et mai 2017 et ayant renseigné un questionnaire ont été inclus. Depuis août 2016, le centre propose à ses consultants une notification par SMS après le dépistage. Les données sont issues de la base de données de la consultation et d'un questionnaire auto-administré rempli à la primo-consultation portant sur les caractéristiques sociodémographiques, les comportements sexuels et le contexte de venue. L'étude des facteurs associés au non-retour a fait appel à des modèles de régression logistique univariée et multivariée.

\section{Résultats}

Parmi les 214 consultants inclus, le taux de non-retour était de $14 \%$ (IC95 \% [10\%-19\%]). Les consultants les plus à risque de non-retour étaient : les moins de 25 ans (ORa : 2,84; IC95 \% : 1,097,41), ceux se percevant moins à risque de contamination par le VIH (ORa : 3,05; IC95 \% : 1,17-7,94), 
ceux n'ayant pas réalisé de dépistage chlamydia/gonocoques par auto prélèvement (ORa : 5,98 ; IC95 \% : 2,03-17,6), les personnes venues accompagnées (ORa : 3,73; IC95 \% : 1,41-9,88). Le taux de non-retour était plus faible chez les consultants ayant accepté d'être notifiés par SMS que ceux ayant refusé, sans différence statistiquement significative ( $12 \%$ vs $20 \%, p=0,10$ ).

\section{Conclusion}

La diversification des modalités de communication avec les consultants (par exemple, appel téléphonique systématique) doit être poursuivie pour diminuer le taux de perdus de vue. Le profil de consultants à risque de non-retour mis en évidence dans cette étude suggère une association entre le non-retour et les représentations des consultants concernant la prévention des IST. Accroître le niveau de littératie en santé sexuelle des individus pourrait permettre de réduire le taux de non-retour dans les centres de dépistage.

\section{Déclaration de liens d'intérêts}

Les auteurs déclarent ne pas avoir de liens d'intérêts. 